Rev. Ivan BODROŽIĆ ${ }^{*}$

\title{
IL CRISTIANESIMO COME RELIGIO DIVINA NEL PENSIERO DI SANT'ILARIO DI POITIERS
}

Sant'Ilario di Poitiers ha vissuto in un momento storico difficile in cui, la crisi nei rapporti tra il potere politico e quello ecclesiastico si era notevolmente aggravata a causa della lotta tra le varie frazioni cristiane che non trovavano pace neanche dopo il concilio di Nicea dove è stata firmata la professione di fede della Chiesa. Da una parte, con la svolta costantiniana, il Cristianesimo era stato riconosciuto come una religione lecita e addirittura era sempre più appoggiata dagli imperatori, dall'altra si rischiava un'interferenza inammissibile del potere civile negli affari interni della Chiesa, perché l'imperatore Costanzo, si era schierato dalla parte degli ariani. Anzi, avrebbe voluto risolvere politicamente il problema teologico imponendo le soluzioni che egli stesso considerava giuste, non tenendo conto che avrebbe minato la verità divina con i compromessi teologici. In questo ambito vogliamo condurre la nostra ricerca teologica sui vari significati del termine religio nelle opere di sant'Ilario che è stato partecipe degli eventi negli anni 50 e 60 del IV secolo. L'intenzione è quella di, a partire dall'analisi dei suoi testi, mettere in risalto il suo modo di intendere la religione e soprattutto vedere quali sono i criteri della sua comprensione delle religioni e della Chiesa, in quanto anch'essa può essere compresa sotto il termine di religione.

\section{IL SIGNIFICATO DEL TERMINE RELIGIO}

Per poter esporre il significato del termine religio negli scritti di sant'Ilario e il modo in cui intende il cristianesimo e la Chiesa Cattolica come la religio divina, prima di tutto va spiegato il concetto di religione. Da principio va detto

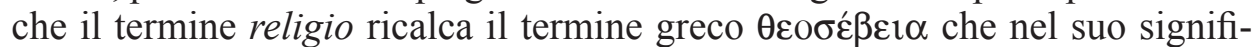
cato classico significa rispetto per gli dei o religiosità ${ }^{1}$. Definito in tal modo ovviamente rappresentava parecchie difficoltà perché tale definizione risulta molto vaga. Tanto è vero che nel periodo precristiano il problema non veniva posto in quanto non ci si poneva in maniera radicale il problema dell'esistenza degli dei né del loro ruolo nella vita dell'uomo, a differenza di ciò che faranno

${ }^{*}$ Rev. Prof. dr. sc. Ivan Bodrožić - Presidente della Cattedra delle Lettere Cristiane e della Dottrina Cristiana, Facoltà Cattolica a Zagreb; e-mail: ivan.bodrozic@gmail.com.

${ }^{1}$ Cf. H.G. Liddel - R. Scott, Dizionario illustrato greco-italiano, Firenze 1993 ${ }^{11}, 588$. 
i cristiani che si porranno effettivamente questo problema, consapevoli che Dio vivo e vero è uno solo. Generalmente la gente praticava un certo rispetto per varie divinità, che comprendeva anche il culto degli dei privati o pubblici (statali). In tale ambiente è stato definito come religioso, ossia pio e devoto nel confronto degli dei, chiunque praticava certi riti con i quali onorava gli dei. Va detto anche che nel latino classico si faceva la distinzione tra la superstizione e la religione, ma come si vedrà in seguito, tale distinzione non è stata molto chiara, netta e felice ${ }^{2}$. Invece il concetto di religio nel senso proprio significava una specie di paura e il culto degli dei che si può chiamare pietà. Di conseguenza la religione poteva significare anche la cura e le cerimonie che si offrono a qualche natura superiore. In modo particolare si riferisce agli stessi riti con i quali si onora la divinità 3 .

Per descrivere i propri sentimenti e atteggiamenti nei confronti di Dio, oppure per designare il proprio culto, $\mathrm{i}$ cristiani non avevano altra parola da usare: usavano questo stesso termine, però non con l'identico significato. Essi cercavano di darne un senso più profondo, ritenuto da loro vero ed autentico. In tal

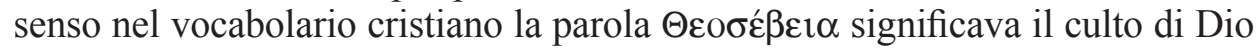
oppure si poteva riferire alla religione intesa nel senso della dottrina e della prassi .Va notato anche che per i cristiani questa parola veniva presa nel senso di ortodossia ossia come espressione di retta fede dei cristiani. Per quanto riguarda il versante della prassi si poteva riferire alla pietà e al retto modo di agire ${ }^{4}$.

Tutto ciò non avviene solamente con gli autori greci, ma si trova anche negli autori latini. Lattanzio ad esempio, uno dei primi autori latini che se n'è occupato, si oppone all'interpretazione ciceroniana del concetto di religio, ritenendo come superstizione sia ciò che i pagani consideravano superstizione sia ciò che consideravano religione, trattando alla stessa stregua tutte e due. Secondo il "Cicerone cristiano" la religione pagana è davvero una superstizione perché chiedeva di adorare gli dei inesistenti. Egli ad esempio intende il cristianesimo come una religione divina o religione di Dio, non solo nel senso che onorava il Dio unico e vero, ma anche in quanto è stato Dio a congiungere gli uomini a se stesso e non sono gli uomini a unirsi a lui con le proprie forze ${ }^{5}$. Quindi Lattanzio è esplicito nell'affermare che il cristianesimo è la vera religione perché ha il suo inizio e la sua fonte in $\mathrm{Dio}^{6}$.

${ }^{2}$ Cf. Cicero, De natura deorum 2, 72. Coloro che trascorrevano le giornate intere a pregare e a far sacrifici perché i loro figli sopravvivessero (superstites essent), Cicerone li riteneva superstiziosi, mentre coloro che riconsideravano e, per così dire, "rieleggevano" tutte le pratiche del culto furono detti religiosi dal verbo relegere.

${ }^{3}$ Cf. A. Forcellini, Lexicon Totius Latinitatis, vol. 4, a cura di G. Perin, Padova 19652, 68-69.

${ }^{4}$ Cf. Lampe, s. 635-636.

${ }^{5} \mathrm{Cf}$. Lactantius, Divinae institutiones 4, 28. In un modo simile anche nel De mortibus persecutorum Lattanzie più volte parla del cristianesimo come della religione di Dio cioè religio Dei (vedi numeri 3, 10 e 31) oppure della divina (num. 11) o santa (num. 24) religione.

${ }^{6} \mathrm{Cf}$. ibidem 4, 4, PL 6, 456: "Fons autem sapientiae et religionis, Deus est: a quo hi duo rivi si aberraverint, arescant necesse est; quem qui nesciunt, nec sapientes esse possunt, nec religiosi”. 


\section{I SIGNIFICATI DEL TERMINE RELIGIO NEGLI SCRITTI DI SANT'ILARIO}

A questo punto, dopo le considerazioni iniziali, passando all'analisi del concetto di religio nel pensiero di sant'Ilario, si denota un uso molteplice del termine religio, ma in coerenza con la tradizione ecclesiastica precedente. Il termine serve per esprimere non qualsiasi tipo di legame religioso, ma quello autentico. Già dai testi di Lattanzio si ravvisa con quanta cautela i cristiani applicano a se stessi questo concetto. Mentre ancora il culto pagano veniva definito religione, i cristiani, a differenza loro, mettevano sempre in evidenza quella differenza specifica che esisteva tra la religione cristiana e le altre religioni: il cristianesimo è la religione di Dio, mentre le altre sono le religioni umane e di conseguenza vengono considerate come superstizioni. Anche se negli scritti di Ilario possiamo individuare vari sensi della parola religio, perché infatti aveva tanti significati, tuttavia dobbiamo osservare che viene usata con coerenza rispetto a ciò che è stato detto finora, sia che si tratti del culto di Dio o di sentimento personale oppure della professione della fede. Possiamo dire che si tratta di differenti facce della stessa realtà, anche se nelle lingue moderne non possono essere tradotte sempre allo stesso modo come religione, ma piuttosto usando anche altri termini: fede, devozione, pietà, senso religioso ecc.

È bene che prendiamo in esame i brani più significativi, tralasciando vari ambiti in cui la parola religio veniva usata in un senso ordinario di pietà o di devozione volendo mettere in risalto i tratti più significativi. Lasciando da parte dei brani "neutri", se possono esserci come tali al di fuori del contesto polemico, e passiamo piuttosto all'analisi dei due ambiti fortemente sentiti nei quali il Vescovo di Poitiers fa vedere la propria punta mordente. Infatti, mentre combatteva ardentemente per la verità cattolica, combatteva anche per una retta e coerente interpretazione di questo termine. Nei suoi testi più polemici Ilario non discute contro la religione pagana, ma si muove in altre due direzioni: da una parte si oppone agli eretici ariani e dall'altra si scaglia contro l'imperatore e il potere imperiale cha dava pieno appoggio proprio a loro. La sua reazione, a due livelli distinti, la ravvisiamo meglio in due delle sue opere: la reazione contro gli ariani si legge nel De Trinitate $^{7}$, mentre quella nei confronti dell'imperatore nel Contra Constantium ${ }^{8}$.

${ }^{7}$ Mi sono servito della traduzione italiana: Ilario di Poitiers, La Trinità I e II, introduzione, traduzione e note a cura di A. Orazzo, Roma 2011. Invece per il testo latino mi sono servito dell'edizione J.P. Migne, PL 10, 9-742.

${ }^{8}$ Traduzione italiana: Ilario di Poitiers, Contro l'imperatore Costanzo, traduzione, introduzione e note a cura di L. Longobardo, Roma, 1997. Per il testo latino mi sono servito dell'edizione J.P. Migne, PL 10, 571-606. 


\section{RELIGIO NELLA POLEMICA CONTRO GLI ERETICI ARIANI}

1. La religiosità falsa degli ariani. Non potendo entrare, in questa sede, nei dettagli dello scontro avvenuto dopo il concilio di Nicea tra gli ariani e i cattolici perché ci avrebbero trascinato lontano dal tema, qui ci limitiamo a dire che la battaglia si combatteva circa la comprensione, la persona e il ruolo di Gesù Cristo. Gli ariani accettavano solo il Padre come Dio, considerando il Figlio la più perfetta delle creature, mentre la Chiesa professava la sua piena divinità e la consustanzialità con il Padre .

Per quanto riguarda invece il tema di religio nel pensiero di sant'Ilario, possiamo osservare che la sua riflessione è condizionata dalla polemica con gli ariani. Non solo la Chiesa, ma anche essi affermavano di stare dalla parte del vero e in tal senso sostenevano di essere autentici cristiani, vantandosi anche di possedere autentica pietà e senso religioso. Lo ravvisiamo chiaramente da vari testi ilariani nei quali ci ha lasciato la testimonianza che gli ariani si arrogavano il diritto di avere la vera religione ossia di possedere l'autentica pietà e devozione di Dio. In uno di questi Ilario, dopo aver commentato alcuni brani dell'Apostolo (cf. Tit 2, 78; 1, 10; 1Tm 2, 7; $2 \mathrm{Tm} 2,17$ ) e dopo aver citato Tit 1,10 , rinfaccia agli ariani:

"Il motivo che mi ha fatto ricordare questa frase dell'Apostolo è il seguente: uomini della mente perversa, ingannevoli nella professione di fede, vuoti nella speranza, viperini nella parola, ci impongono la necessità di contraddirli, perché sotto l'apparenza di pietà (per speciem religionis) insinuano negli ascoltatori semplici insegnamenti letali, modi malsani di intendere, volontà corrotta"10.

Gli ariani, quindi, lasciavano l'impressione di possedere l'autentico senso religioso e di combattere per le proprie idee spinti proprio da un tale senso. Ilario invece tale senso lo ritiene falso e simulato e cerca di smascherarlo ad ogni costo. Così vediamo che anche in un altro testo nel libro decimo egli stigmatizza tale atteggiamento ipocrita ed insincero da parte di coloro che non sono in grado di ascoltare Dio e si inventano una dottrina più idonea ai propri desideri piuttosto che riflettere su ciò che egli ha rivelato:

"Quale profitto c'è in una dottrina che cerca ciò che piace anziché ciò che si deve insegnare? O quale venerazione per la dottrina c'è, quando non si desidera ciò che si deve insegnare, e invece si mette insieme una dottrina adatta ai

\footnotetext{
${ }^{9}$ Per chi volesse saperne di più sulle dispute teologiche di questo periodo, indico alcuni studi: M. Weedman, The Trinitarian Theology of Hilary of Poitiers, Leiden - Boston 2007; C.L. Beckwith, Hilary of Poitiers on the Trinity, Oxford 2008; L.F. Ladaria, La cristologia de Hilario de Poitiers, Roma 1989; S. Petri, Introduzione a Ilario di Poitiers, Brescia 2007.

${ }^{10}$ Hilarius Pictaviensis, De Trinitate 8, 2, PL 10, 237: "Ac mihi quidem ea nunc commemorandae huius sententiae apostolicae fuit causa, quia homines mente perversi, et professione fallaces, et spe inanes, et sermone viperei, contradicendi nobis necessitatem imponunt: dum lethales doctrinas, et morbidas intelligentias, et corruptas voluntates, simplicitati audientium per speciem religionis insinuant".
} 
desideri? Ma queste istigazioni degli spiriti seduttori abbondano e rafforzano le menzogne di una pietà insincera" 11 .

Come vediamo, Ilario insiste che non ci può essere un vero profitto della dottrina (religio doctrinae) se non si accetta quella dottrina che Dio ha voluto rivelare. Gli ariani, insistendo a credere ciò che vogliono invece di sottomettersi alla dottrina di Dio rimangono fortificati nella menzogna della loro falsa pietà (simulatae religionis falsaloquia confirmant). Ilario ritiene che le conseguenze di tale falsa religiosità sono gravi, come mette in evidenza nel resto dello stesso brano:

"Difatti, all'eclissarsi della fede segue una ipocrisia ingannevole, in modo che almeno nelle parole ci sia quella pietà che la coscienza ha smarrito. E rendono empia la stessa pietà simulata con ogni genere di sofismi, corrompendo la rettitudine della fede con gli insegnamenti di una dottrina ingannatrice. Si tratta di una dottrina architettata in conformità alle inclinazioni dei loro desideri più che alla fede evangelica. Con gli orecchi stimolati da prurito, essi sono solleticati dal piacere intollerante di udire una predicazione nuova, conforme ai propri desideri; del tutto lontana dall'ascolto della verità, si affidano totalmente alle favole. Incapaci sia di esprimere che di ascoltare le cose vere, cercano di coprire con un'apparenza di verità le cose che dicono"12.

In questo testo troviamo le obiezioni principali che Ilario muove contro gli ariani rimproverando loro una pietà (religio) falsa e simulata. Se d'altra parte vogliamo trarre conclusioni positive su come Ilario vede la religiosità autentica con delle caratteristiche inoppugnabili, possiamo concludere così: La vera religiosità non è solo a parole, ma è sentita nel profondo della coscienza e corrisponde alle profondità più intime dell'essere. Per niente può essere simulata e difesa con parole menzognere, ma piuttosto deve poggiare sulla santità della fede. Invece di seguire i desideri delle proprie inclinazioni, si regola sulla fede evangelica. E alla fine si distingue per il fatto di mantenere la fedeltà alla verità rivelata, invece di voler introdurre una predicazione nuova, coperta con un'apparenza di verità.

Ogni atteggiamento di pietà religiosa che si scostava da tali criteri secondo Ilario non poteva essere chiamato pietà (religio), ma piuttosto empietà. Al di là di questi giudizi di Ilario sulla pietà degli ariani, che secondo lui risultava

${ }^{11}$ Ibidem 10, 2, PL 10, 345: "Qui enim doctrinae profectus est, placita magis quam docenda conquirere? Aut quae doctrinae religio est, non docenda desiderare, sed desideratis coacervare doctrinam? Sed haec seducentium spirituum incentiva suppeditant, et simulatae religionis falsiloquia confirmant".

${ }^{12}$ Ibidem, PL 10, 345-346: "Sequitur enim fidei defectionem hypocrisis mendax, ut sit vel in verbis pietas, quam amiserit conscientia. Et ipsam quidem simulatam pietatem omni verborum mendacio impiam reddunt, falsae doctrinae institutis corrumpentes sanctitatem fidei: dum secundum desideria studiorum potius, quam secundum evangelicam fidem, coacervata doctrina est. Auribus enim prurigine incitatis, dum per audiendi impatientem oblectationem sub novella desiderii sui praedicatione scalpuntur, ipsi penitus ab auditu veritatis alieni, totos se fabulis destinant: ut his, quae loquantur, veritatis speciem acquirant, dum quae vera sunt et loqui et audire non possunt". 
falsa, ci sono anche altre difficoltà di ordine teologico. Anche gli ariani si vantavano di possedere la verità, bisognava, dunque, dimostrare chi stava dalla parte del vero. A questo punto, per dimostrare chi aveva ragione, si dovevano introdurre delle prove.

2. La dimostrazione della religiosità autentica. Una delle prime prove concerneva l'esegesi della Sacra scrittura. Ilario ci mette un grande sforzo per spiegare vari passi scritturistici in coerenza con la tradizione della Chiesa e nello stesso tempo smascherando e correggendo l'esegesi erronea degli ariani, che pure si appellavano ai testi biblici per giustificare le loro dottrine. Secondo Ilario, essi non erano in grado di capire bene le Scritture perché la loro finta devozione fondata su una dottrina fallace, alterava la comprensione delle parole scritturistiche (dicti tamen intelligentiam fallacis doctrinae religio ementita corrumperet) ${ }^{13}$. Quindi, partendo da un sbagliato spirito di religiosità sono stati condizionati e impediti ad afferrare bene i misteri compresi nei testi sacri.

Comunque Ilario non si è limitato semplicemente a richiamarsi ai soli testi scritturistici, perché anche gli ariani si richiamavano ad essi a sostegno delle proprie idee, ma la sua argomentazione doveva contenere anche altre dimostrazioni contro la sfida degli ariani. Tale prova consiste nell'insistere sulla fedeltà alla tradizione apostolica accusando gli ariani di introdurre una nuova predicazione conforme ai loro desideri e non al Vangelo. Anzi, in altri testi ancora è molto esplicito nell'affermare che si sono scostati dalla predicazione apostolica che la Chiesa trasmetteva fedelmente dall'inizio. Nel libro ottavo si esprime così:

"Tralasciando l'integrità della predicazione apostolica, fanno in modo che per essi il Padre non sia padre, Il Figlio non sia figlio, Dio non sia dio e la fede non sia fede" $" 14$.

Lo stesso fa anche nel libro decimo quando discute con gli ariani sul come e perché della passione del Signore e delle debolezze della carne. A suo avviso, se si ripudia la dottrina apostolica, che ci trasmette il vero senso della sua passione, viene cambiato anche l'atteggiamento religioso di fronte a Dio:

"Se invece nel mistero della passione è annunciato il contrario di questo, quale follia - chiedo - si trova nel cambiare il senso del vincolo religioso ripudiando la dottrina della fede apostolica, ad impadronirsi di tutto questo, ritenendo obbrobrio di una natura debole ciò che è un atto di volontà e un mistero, ciò che è potenza, coraggio e trionfo?"'15

${ }^{13}$ Ibidem 9, 1, PL 10, 280. Così ad esempio per tutta una serie di testi del Vangelo che parlano dell'unità del Padre e del Figlio (cf. Gv 14, 9; 10, 38; 10, 30; 14, 15) gli ariani spiegavano come una unione e concordia di volontà piuttosto che come uguaglianza di natura.

${ }^{14}$ Ibidem 8, 2, PL 10, 237: "Id apud eos praetermissa apostolicae praedicationibus sinceritate agentes, ne Pater pater sit, ne Filius filius sit, ne Deus deus sit, ne fides fides sit".

${ }^{15}$ Ibidem 10, 48, PL 10, 382: "Sin antem haec e contrario in sacramento passionis praedicantur; 
A causa di tutto ciò egli parlerà degli ariani come di nuovi riformatori della fede apostolica (novi apostolicae fidei emendatores) ${ }^{16}$.

È ovvio che tale affermazione contiene una pesante accusa contro la loro teologia e il loro comportamento religioso. Ilario è convinto che gli ariani, rifiutandosi di riconoscere Gesù Cristo come vero Figlio di Dio consustanziale al Padre, sotto il nome della fede demoliscono la fede ${ }^{17}$. Come fede vera qui è intesa la fede della Chiesa cattolica professata con coerenza dall'inizio a partire dagli apostoli, testimoni privilegiati del Signore Gesù. In tal senso Ilario invita gli ariani a riconoscere l'intelligenza della fede e non distorcere la retta professione. Essi, infatti, alle parole della professione della fede della Chiesa davano la loro interpretazione erronea:

"Impara a comprendere la tua confessione di fede, e conosci l'unico e vero Dio. Ti impadronisci infatti di quanto professa la nostra fede a vantaggio della tua empietà, e neghi ciò che è vero pur non negandolo. In tal modo lusinghi la stolta sapienza del mondo, per demolire la verità sotto l'apparenza pretestuosa della verità. Confessi un unico vero Dio, per negare l'unico vero Dio. La tua professione di fede passa per devota proprio perché è empia; passa per vera, proprio perché è falsa"18.

Gli ariani quindi, secondo l'interpretazione di Ilario, non tenendo la retta fede possono solo fingere di essere devoti (fingere religionem) ${ }^{19}$, ma in realtà non lo sono. Ecco perché egli considera l'arianesimo come una falsa religione che si è avvalsa del nome di vera (ementito nomine verae religionis) spargendosi per tutte le provincie dell'Impero romano ${ }^{20}$. Secondo Ilario il comportamento degli ariani, dal punto di vista religioso, è veramente perverso, perché sotto il pretesto del monoteismo, confessato pure dai cattolici, e in apparenza con molta devozione (religiose) verso il Padre, negano la divinità del Figlio. Ilario, dopo aver analizzato la loro professione di fede, sostiene che non fanno altro

quis, rogo, furor est, repudiata doctrinae apostolicae fide mutare sensum religionis, et totum hoc ad contumeliam imbecillis rapere naturae, quod et voluntas est et sacramentum, quod et potestas est et fiducia et triumphus?".

${ }^{16}$ Ibidem 12, 3, PL 10, 435.

${ }^{17}$ Cf. ibidem 5, 10, PL 10, 135: "Quid sub religionis nomine religionem evertis?"

${ }^{18}$ Ibidem 5, 25, PL 10, 146: "Disce confessionis tuae intelligentiam, et unum ac verum Deum cognosce, ut unum ac verum Deum pie praedices. Rapis enim ad impietatem tuam religionis nostrae professionem: et quod est negas, dum quod est non negas. Sic stultam sapientiam fallis, ut veritatem perimas specie veritatis. Unum verum Deum confiteris, ut unum verum Deum deneges. Professio enim tua sic putatur pia, quod magis impia est; sic vera quod falsa est. Sic a te unus et verus Deus praedicatur, ut non sit".

${ }^{19}$ Ibidem 7, 1, PL 10, 199.

${ }^{20}$ Ibidem 6, 1, PL 10, 158. Si può vedere anche 6, 10, PL 10, 164 dove Ilario sostiene che la follia eretica finge delle preoccupazioni insensate sotto falsa apparenza della fede (ementita religionis specie), come anche il testo citato del De Trinitate 9, 1 dove si trova la stessa accusa contro la religione eretica di essere una religione falsa e simulata (fallacis doctrinae religio ementita). 
che servirsi dei principi della religione (religionis usa principiis) per insegnare la loro empietà e per negare il mistero della vera fede:

"L'eresia infatti nega, furiosa qual è, il mistero della vera fede; e per insegnare la sua empietà si serve dei principi della religione, quando - come è detto nei libri precedenti - inizia ad esporre così la sua incredulità: Conosciamo un solo Dio, solo increato, solo eterno, solo senza inizio, solo vero, solo immortale, solo ottimo, solo potente. A questo infatti, conduce l'inizio della retta confessione di fede, da essi rivendicato, quando dice: Un solo Dio, e un solo increato, e un solo senza inizio, e così possono introdurre empiamente altre affermazioni mediante queste che a parole devotamente ostentano. Difatti, dopo molti altri particolari espressi circa il Figlio con una confessione simile di fede simulata, l'eresia ha aggiunto: Creatura perfetta di Dio, ma non come una delle creature, opera [sua] ma non come le altre opere"21.

E per concludere la nostra analisi dei testi indichiamone un altro nel quale Ilario fa vedere il proprio impegno circa la verità della fede, consapevole che la religione senza la verità non ci può essere. La verità è un elemento costitutivo della religione, mentre, dall'altra parte, l'empietà si distingue per la falsità:

"Sarebbe una fatica molto dura e gravosa proteggere la verità della fede contro la falsità empia, se negli eretici alla misura dell'audacia corrispondesse la misura della prudenza. Ma, fortunatamente, la spinta interiore all'empietà viene da una mancanza di prudenza! E perciò, pur essendo facile rispondere alla stoltezza, è tuttavia difficile correggere gli stolti; prima perché non si cercano le ragioni per comprendere; poi perché non si accettano, anche se comunicate da chi le ha comprese. Ma se alcuni sono trattenuti nell'errore dal timore di Dio e dall'ignoranza delle motivazioni, e non da una volontà di empietà associata a un modo stolto di pensare, spero che questi si sentano inclini ad emendarsi, dal momento che la dimostrazione chiara della verità fa emergere la stoltezza dell'empietà'’22.

${ }^{21}$ Ibidem 6, 4, PL 10, 133: "Negat enim, negat furens haeresis sacramentum verae fidei, ad impietatis suae doctrinam religionis usa principiis, cum infidelitatis suae expositionem, ut superioribus libris continetur, ita coepit: «Novimus unum Deum solum infectum, solum sempiternum, solum sine initio, solum verum, solum immortalitatem habentem, solum optimum, solum potentem». Ad id enim usurpatum proficit piae confessionis exordium, quo ait: «Unum Deum, et solum infectum, et solum sine initio»: ut per haec, quae religiose verbis ostentarentur, caetera impie subderentur. Nam post multa alia, quae de Filio pari quoque simulatae religionis professione protulerat, subjecit: «Creaturam Dei perfectam, sed non sicuti unum de creaturis; facturam, sed non sicuti caeterae facturae»".

${ }^{22}$ Ibidem 6, 15, PL 10, 143: "Gravis et multae difficultatis labor esset veritatem religionis adversum falsitatis impietatem tueri, si quantum audet impietas, in tantum consuleret prudenter. Sed bene, quod irreligiositatis voluntas ex inopia prudentiae est. Et idcirco cum facilis sit adversum stultitiam responsio, emendatio tamen difficilis stultorum est: per quam primum et ratio intelligentiae non quaeritur, et deinceps ab intelligente intimata non capitur. Sed si quos timor Dei et intelligentiae ignoratio, non impietatis voluntas per stultitiae sensum detinuerit in errore, spero ut ad emendationem proclives sint; cum impietatis stultitiam absolute veritatis sit demonstratio proditura". 
A tale conclusione, come abbiamo visto nell'analisi sopra riportata, lo spingono motivazioni interne su ciò che egli intendeva per religione. Di conseguenza, dal punto di vista teologico, gli ariani in quanto seguivano una religione di principi falsi non si potevano presentare come una vera religione né aspettarsi l'appoggio dell'imperatore. L'eresia non possedeva i principi richiesti per poter essere una religione. Tali principi vengono tratti dalla retta confessione di fede. I principi della fede non sono provvisori, a piacere di singole persone o gruppi, ma sono fissati da Dio stesso, ricevuti e custoditi dalla Chiesa fondata sugli apostoli che ne erano testimoni autentici.

\section{LA REAZIONE CONTRO IL POTERE STATALE}

Il secondo ambito in cui sant'Ilario oppone le sue convinzioni religiose è l'ambito dello scontro con l'imperatore Costanzo che pure nel 356 ha firmato la sua condanna all'esilio ${ }^{23}$. Tralasciando le vicende storiche che hanno portato a questa condanna, ci concentriamo sul nostro argomento dando per sommi capi la cornice in cui è avvenuto lo scontro tra Ilario e l'imperatore Costanzo. L'imperatore si era schierato dalla parte ariana, o più precisamente ha insistito sulla soluzione teologica della controversia dando l'avvio e l'appoggio al gruppo dei cosiddetti ome ${ }^{24}$. L'opera in cui attestiamo meglio la risposta di sant'Ilario è il Contra Constantium nella quale spietatamente denuncia la filoariana politica imperiale. Qui metto in evidenza alcuni testi ai quali ho già richiamato attenzione in un articolo precedente ${ }^{25}$, ma vale la pena riproporli anche in questa analisi.

Ciò che ha detto nel De Trinitate in modo generale contro gli ariani, nel Contra Constantium lo riespone in modo concreto e sintetico contro la persona dell'imperatore Costanzo. Secondo Ilario l'imperatore inganna presentandosi con il falso nome (cristiano) e così consuma l'empietà sotto l'apparenza della religione. Si presenta come predicatore di Cristo, invece soffoca la fede in Cristo ${ }^{26}$. Non solo l'imperatore, secondo il Nostro, presenta un'apparente pietà, ma si serve di una scaltra professione di fede (professio religionis) ${ }^{27}$.

Ma dopo le osservazioni sulla religiosità dell'Imperatore, vanno esaminati i testi nei quali Ilario giustifica le proprie vedute e condanna le posizioni di

${ }^{23}$ Per saperne di più si può vedere: P.C. Burns, Hilary of Poitiers' Road to Béziers: Politics or Religion?, JECS 2 (1994) 273-289; T.D. Barnes, Hilary of Poitiers on His Exile, VigCh 46 (1992) 129-140. Sui motivi della politica di Costanzo si può vedere: T.D. Barnes, Athanasius and Constantius. Theology and politics in the constantinan empire, London 1993, 165-175.

${ }^{24}$ Cf. M. Simonetti, Omei, NDPAC II 3599-3600.

${ }^{25} \mathrm{Cf}$. I. Bodrožić, Non recipit mendacium veritas, nec patitur religio impietatem. La reazione di Ilario di Poitiers contro la legislazione dell'imperatore Costanzo, in: "Lex et religio". XL incontro di studiosi dell'antichità cristiana (Roma, 10-12 maggio 2012), SEA 135, Roma 2013, 521-533.

${ }^{26}$ Cf. Hilarius Pictaviensis, Contra Constantium 8, PL 10, 585: "Subrepis nomine, blandimento occidis, specie religionis impietatem peragis, Christi fidem Christi mendax praedicator exstinguis".

${ }^{27}$ Ibidem 21, PL 10, 596: "Quae ergo callida religionis tuae professio est, similem secundum Scripturas Patri Filium dicere; cum ad imaginem et similitudinem Dei homo tantum factus sit?" 
Costanzo. In un testo Ilario afferma che la religiosità ariana non è autentica, ma empia e fallace. Ilario ricorda che così come non c'è niente in comune tra la verità e la menzogna, non c'è neanche niente in comune tra la religione e l'empietà: "La verità non ammette menzogna, la religione non sopporta l'empietà"28. Ovviamente stigmatizzava la religiosità ariana come empia proprio perché non è stata in grado di ricevere la verità di Dio, ma si fondava sulla menzogna delle idee umane. Insistendo sul binomio verità-religione Ilario ha riproposto, anche contro l'Imperatore, il criterio di cui si era avvalso già nel De Trinitate.

Per completare la nostra analisi sono importanti altri due testi molto significativi e più polemici di questo testo sopra riportato. Scagliandosi contro l'imperatore che assume il ruolo del promotore dell'arianesimo, Ilario avverte:

"Considera tu stesso se, professando a parole la fede nel nome del Signore, nei fatti compi la volontà di Dio Padre. Lui afferma chiaramente: «Questi è il Figlio mio prediletto, nel quale mi sono compiaciuto», e tu stabilisci che non c'è né figlio né padre, ma solo nomi di adozione, appellativi esteriori e, novello persecutore della religione divina, introduci un Dio che dice tutte cose false su se stesso"29.

Tra l'altro, vediamo che Ilario gli indica la fede della Chiesa, che egli non rispetta e addirittura perseguita. Facendosi persecutore della Chiesa, secondo Ilario, l'imperatore è diventato persecutore non di una religione qualsiasi, ma della religione divina. Da tale frase traspare la sua convinzione su ciò che è la Chiesa e sul perché la ritiene unica e vera religione. Le sue convinzioni di fede si fanno vedere ancora più chiaramente in un brano molto sintetico in cui Ilario elenca a Costanzo le ragioni teologiche in base alle quali lo ritiene avversario della religione divina:

"Intendi la santa comprensione delle parole, intendi l'immutabile costituzione della Chiesa, intendi la fede professata da tuo padre, intendi la fiduciosa certezza della speranza umana, intendi la coscienza del popolo che condanna eresia e comprendi che sei l'avversario della religione divina, il nemico della memoria dei santi e un ribelle contro la retta fede di tuo padre" ${ }^{30}$.

Ilario ritiene, quindi, che dovrebbero essere più che convincenti i maggiori argomenti teologici, perché l'imperatore si converta: il retto modo di comprendere la Scrittura, la presenza e la testimonianza della Chiesa nella sua co-

${ }^{28}$ Ibidem 25, PL 10, 600: "Non recipit mendacium veritas, non patitur religio impietatem".

${ }^{29}$ Ibidem. 9, PL 10, 585: "At vide tu, si praeferens nomen Domini in verbis, voluntatem efficias Dei patris in rebus. Clamat ille: Hic est filius meus dilectus, in quo mihi bene complacui (Mt 3, 17): et tu decernis non esse filium, neque esse patrem; sed adoptionis nomina, externas nuncupationes, et simulantem de se omnia Deum novus hodie religionis divinae persecutor inducis".

${ }^{30}$ Ibidem 27, PL 10, 602: "Audi verborum sanctam intelligentiam, audi Ecclesiae imperturbatam constitutionem, audi patris tui professam fidem, audi humanae spei confidentem securitatem, audi haereticae damnationis publicum sensum, et intellige te divinae religionis hostem, et inimicum memoriis sanctorum, et paternae pietatis haeredem rebellem". 
stituzione immutabile, la professione di fede di Nicea, le sane speranze umane e la coscienza del popolo (sensus fidelium). Un'altra volta vediamo come il Vescovo di Poitiers, volendo scuotere la coscienza dell'Imperatore, gli sottolinea che è l'avversario della religione divina. Ilario sperava che l'Imperatore, se in lui fosse rimasto del senso cristiano, avrebbe accettato come vincolante in coscienza la verità di fede cristiana dimostrata con tale rigore teologico ${ }^{31}$.

Nonostante l'impegno dell'imperatore Costanzo nelle questioni della religione fosse notevole, tuttavia non era sufficiente perché egli non partiva dai sani presupposti teologici in materia di religione. Ilario fa vedere che egli non ha capito il contributo che ha apportato la Chiesa in materia religiosa imponendosi come una religione divina in coerenza con tradizione secolare. Se l'imperatore avesse capito la portata di tale verità avrebbe reagito diversamente. Invece con il suo operare aveva messo la Chiesa fuori la legge.

L'imperatore Costanzo sapeva, e questo è il suo merito, che la religione non poteva più essere definita in termini neutrali. Da tale consapevolezza scaturisce anche il suo impegno in materia di religione, ma egli stesso non ha usato i criteri radicali per capire che cosa è la vera religione, ossia quella divina, né ha usato i mezzi giusti degni di tale religione. Non ha rispettato la logica che era intessuta nella stessa fede rivelata, lasciando alla Chiesa di definire la fede da professare, ma ha provato lui ad esserne arbitro appoggiando le professioni degli omei. Se avesse creduto che la vera religione coincideva con la retta fede, definita in coerenza con la verità divina e rivelata, si sarebbe comportato diversamente. In queste nuove circostanze avrebbe capito che la coerenza di ragionamento di fede rimane la stessa e che la Chiesa non poteva rinunciare ad esse, anche se correva il rischio di finire al bando e un'altra volta di essere dichiarata illecita.

Ilario e gli altri difensori dell'ortodossia cattolica dovettero impegnarsi per difendere i diritti e l'autonomia della Chiesa nel campo della religione, consapevole della verità rivelata che lo autorizzava a farlo. Proprio perché la Chiesa possedeva e annunciava la verità divina, essa aveva il diritto di essere considerata vera religione, come abbiamo visto chiaramente nei testi ilariani.

A partire da tali dati, invece di essere vincolata, oppressa e sottomessa alle leggi imperiali, la religione cristiana, in quanto religione rivelata e vera, si libera da vincoli inaccettabili diventando misura di ogni intervento legislativo. Detto in termini diversi, la legislazione imperiale avrebbe dovuto tener conto del fatto che la Chiesa non avrebbe potuto rinunciare alla verità rivelata per la quale aveva una diretta responsabilità davanti a Dio. In nome di tale verità la

${ }^{31}$ In un altro brano nel suo Ad Constantium (II 4, PL 10, 565-566) Ilario ha provato in modo simile a risvegliare la sua coscienza appellandosi alla solenne professione della fede battesimale in cui c'è la confessione della Trinità di cui niente può essere ambiguo né ammette novità contrarie al retto senso di fede. Purtroppo, secondo lui, ci sono alcuni che spinti o dalla presunzione o dalla superficialità o dall'errore, accettano e confessano solo in parte l'immutabile costituzione della dottrina apostolica e dall'altra parte osano uscire fuori dal suo ambito. 
Chiesa, consapevole di essere la religione vera, non avrebbe permesso neanche all'imperatore, costi quel che costi, di modificarne i contenuti. Se l'imperatore voleva essere un pio cristiano e un legislatore onesto che vara le leggi con le quali si regola anche la presenza della religio in una società, avrebbe dovuto innanzitutto informarsi confrontandosi con l'insegnamento della Chiesa e conformandosi alla verità divina. Se l'imperatore Costanzo avesse riflettuto sulla storia della Chiesa avrebbe capito che essa, in quanto religione divina, si regola anzitutto sulle leggi divine, pur nutrendo il rispetto per la legislazione civile nell'ambito della sua competenza, ossia in tutto ciò che non compromette la verità rivelata.

Se fino alla svolta costantiniana esisteva il concetto di religio licita et religio illicita, nel pensiero e nei testi di sant'Ilario queste distinzioni spariscono, anche se non del tutto. Si insiste ormai di più sulla distinzione tra la religione vera e quella falsa, ma il rischio era che l'Imperatore, appoggiando la frazione degli ariani omei, metta fuori legge la Chiesa cattolica considerandola un'altra volta come illecita. Grazie ai santi dottori, disponibili anche a vari sacrifici, è stata lasciata la testimonianza che la religione vera può essere una sola, cioè quella divina, conforme alla verità rivelata. Quelle false, tra le quali non c'è solo la religione pagana, ma vengono enumerate anche le religioni eretiche, non conoscono tale verità, non possiedono tale consapevolezza e non praticano la vera pietà nei confronti di Dio.

\section{$* * *$}

Nel periodo in cui visse Ilario si presenta un aspetto nuovo nel trattare la religione. Mentre nel tempo di transizione lo scontro primario era tra coloro che onoravano il vero Dio (cristiani) e coloro che adoravano varie divinità (pagani), adesso c'è il contrasto e lo scontro tra vari gruppi cristiani, e più precisamente tra i cattolici e gli ariani. Tutti e due i gruppi si chiamavano cristiani e, pure, si contendevano il titolo di vera religione. Il gruppo ariano fu avvantaggiato perché apertamente appoggiato, se non addirittura capeggiato dallo stesso imperatore Costanzo che perseguitava i vescovi che professavano la fede cattolica così che, la Chiesa rischiava di diventare una religio licita come una volta nell'Impero romano.

Questa contesa tra le due frazioni provocava la domanda su quale di esse avesse ragione e quale fosse, di conseguenza, la religione vera. La Chiesa cattolica, come ci testimonia sant'Ilario, ha sempre insistito in questo periodo sul significato autentico della parola religio, chiedendo che la Chiesa cattolica venga riconosciuta dall'imperatore come quella religio vera. Secondo il Vescovo di Poitiers l'unica religio vera a pieno titolo, e quindi dovrebbe essere riconosciuta come tale, è la religione rivelata da Dio, alla quale attribuisce l'appellativo di religione divina. La liceità, alla quale Ilario si appella in maniera indiretta, le proviene da Dio stesso, perché egli l'ha iniziata sulla terra e l'ha autorizzata ad 
annunciare la verità divina. Essa è l'unica religione che è lecita nel senso pieno della parola perché viene da Dio, che è il suo autore. Essa è lecita perché Dio l'ha instaurata e l'ha legittimata sulla terra e nessun potere imperiale la può delegittimare e destituire da tale ruolo. La Chiesa, quindi, attribuisce a se stessa il concetto di religio in maniera assai particolare, a pieno titolo e diritto, e assume questo ruolo, convinta di possederne e riprodurne la pienezza.

Ilario ci fa vedere che il vero atteggiamento religioso non è un atteggiamento formato in base alle convinzioni umane, ma in forza di ciò che Dio ha rivelato. Ecco perché egli reagì con forza contro gli abusi del potere imperiale, convinto che, nei confronti della Chiesa, un imperatore non può comportarsi da padrone assoluto, ossia non può trattare la Chiesa alla stregua della religione pagana. Se il cristianesimo, o meglio la Chiesa, si sentiva come unica religio licita davanti a Dio, allora è Dio il suo padrone assoluto al quale bisogna obbedire. Se la Chiesa poi ha ricevuto da Dio stesso le istruzioni su come adorarlo e onorarlo non in un modo umano, ma in un modo divino, essa non ne poteva fare a meno, anche se doveva contrastare l'imperatore stesso.

\section{CHRZEŚCIJAŃSTWO JAKO BOSKA RELIGIA W MYŚLI HILAREGO Z POITIERS}

\section{(Streszczenie)}

Święty Hilary z Poitiers $(† 367)$ żył w trudnym momencie historycznym, w którym panował kryzys w stosunkach władzy politycznej i kościelnej. Z jednej stony dziedzictwo Konstantyna Wielkiego w postaci uznania chrześciajństwa za religię państwową, $\mathrm{z}$ drugiej zaś pokusy ingerencji włądzy cywilnej w wewnętrzne sprawy Kościoła przez jego nastepców. Zaistniałą sytuację pogarszały znacznie walki między różnymi frakcjami chrześcijan, którzy nie mogli nadal osiagnąć pokoju i zgody w kwestaich religijnych nawet pomimo tego, że na Soborze Nicejskim, zostało ogłoszone wyznanie wiary Kościoła.

Cesarz Konstancjusz, sprzyjający arianon, w rzeczywistości chciał politycznego rozwiązania problemu teologicznego nie uwzględniajac jednak faktu, że kompromis religijny podważy prawdę o Bogu. Na kanwie tych wydarzeń zasadniczą kwestią stanie się precyzyjne określenie pojecia religii prawdziwej. Autor artykułu podjął badania nad różnymi znaczeniami terminu religio w pismach Biskupa Poitiers. Analiza tekstów pozwoliła na zaprezentowanie jego rozumienia religii, a zwłaszcza, wskazanie kryteriów określających zarówno religię jak i Kościół, który również może być rozumiany pod pojęciem religii. Jak konkluduje Autor dla św. Hilarego jedyną, prawdziwą religią jest ta, która została objawiona przez Boga, On sam ją zapoczątkował na ziemi. Dodatkowo religia ta jest zgodna z prawem, ponieważ pochodzi od Boga, który jest jego autorem. Hilary pokazuje wyraźnie, że prawda religijna nie ma prowieniencji ludzkiej, ale jest prawdą ze względu na to, co objawił Bóg. Stąd też postulaty Hilarego pod adresem cesarza, który nie może działać w Kościele jako absolutny pan i traktować Kościoła jako 
religii pogańskiej. Kościół jest depozytariuszem prawdy otrzymanej od Boga jak również wskazówek odnośnie do właściwego i należnego Bogu kultu i nie może odejsć od tego nawet za cenę konfliktu z cesarzem.

Parole chiave: Sant' Ilario di Poitiers, il Cristianesimo, religio divina, la Chiesa. Słowa kluczowe: Św. Hilary z Poitiers, chrześcijaństwo, boska religia, Kościół. 